\title{
OSTEOMA DE SEIO FRONTAL EM PACIENTE JOVEM - UM RELATO DE CASO
}

\section{ARTIGO ORIGINAL}

OLIVEIRA, Ronan Djavier Alves ${ }^{1}$

LOTUFO, Fernanda Botti ${ }^{2}$

RIBEIRO, Stéfani Pellissari da Costa ${ }^{3}$

ESPÓSITO, Mario Pinheiro ${ }^{4}$

BOTTI, Anderson Santos 5

PLACHESKI, Ana Carolina Galindo ${ }^{6}$

PASSOS, Fabio Manoel dos ${ }^{7}$

OLIVEIRA, Ronan Djavier Alves. Et al. Osteoma de seio frontal em paciente jovem - Um relato de caso. Revista Científica Multidisciplinar Núcleo do Conhecimento. Ano

1 Residente em Otorrinolaringologia e Cirurgia Cérvico-Facial, Graduação em Medicina pela Universidade de Cuiabá - UNIC.

${ }^{2}$ Acadêmica de Medicina do Centro Universitário de Várzea Grande- UNIVAG.

${ }^{3}$ Acadêmica de Medicina do Centro Universitário de Várzea Grande- UNIVAG.

${ }^{4}$ Doutor em Otorrinolaringologia pela Faculdade de Ciências Médicas da Santa Casa de São Paulo.

${ }^{5}$ Otorrinolaringologista titulado na ABORL-CCF.

${ }^{6}$ Residente em Otorrinolaringologia e Cirurgia Cérvico-Facial; Graduada em Medicina pela Universidade de Cuiabá - UNIC.

7 Residente em Otorrinolaringologia e Cirurgia Cérvico-Facial; Graduação em Medicina pela Universidade Federal do Paraná - UFPR. 
05, Ed. 09, Vol. 04, pp. 157-162. Setembro de 2020. ISSN: 2448-0959, Link de acesso: https://www.nucleodoconhecimento.com.br/sem-categoria/osteoma-de-seio

\section{RESUMO}

O osteoma do seio frontal é a neoplasia óssea benigna que mais acomete os seios da face. Este apresenta crescimento lento e potencial de crescimento ilimitado, podendo deslocar estruturas adjacentes e ocasionar distúrbios na órbita, endocrânio e fossas nasais. Possui ainda discreta predominância no sexo masculino, nas $3^{\underline{a}}$ e $4^{\underline{a}}$ décadas de vida e o indivíduo, habitualmente, mantém-se assintomático. No relato de caso, demonstramos a importância do diagnóstico precoce, o qual pode ser justificado pelo caráter progressivo e súbito da lesão. Quanto a análise epidemiológica, estudos apontam a incidência de osteoma de seio frontal maior entre indivíduos na segunda década de vida, corroborando com a paciente relatada.

Palavras-chave: Osteoma, seio frontal, tumor.

\section{INTRODUÇÃO}

O osteoma é a neoplasia óssea benigna que mais acomete os seios da face, caracterizada por um crescimento lento e geralmente assintomático. A evolução do tumor pode ocasionar distúrbios na órbita, endocrânio e fossas nasais (BALIEIRO et al., 2004), apresentando sintomatologia variável. O osteoma do seio frontal é mais frequente e apresenta discreta predominância no sexo masculino, sendo mais incidente nas $3^{\mathrm{a}}$ e $4^{\mathrm{a}}$ décadas de vida (OLIVEIRA et al, 2002) (FERREIRA; 1992). Quanto ao tratamento, a cirurgia é indicada apenas nos pacientes sintomáticos, sendo a via de acesso via externa supraciliar ou coronal a principal abordagem (TIAGO et al., 2002). Neste trabalho, os autores relatam caso de paciente feminina jovem que, diferentemente da evolução lenta descrita pela literatura, apresentou rápido progresso do quadro de osteoma de seio frontal. 


\section{RELATO DE CASO}

Paciente M.C.O. de 19 anos, sexo feminino, vem ao consultório em Março de 2019, encaminhada pelo oftalmologista devido achado de exame de ressonância magnética do crânio realizada no mesmo período. A mesma apresentava queixa de abaulamento em região frontal da face à esquerda que teve inicio em Janeiro do mesmo ano, que progrediu rapidamente, de inicio assintomático, progredindo em 4 meses com cefaleia, sem outros sintomas associados, história familiar pregressa ou outras comorbidades. $\mathrm{Na}$ consulta com o oftalmologista, foi solicitado exame de ressonância magnética das órbitas para avaliação do quadro. Este exame radiológico teve como conclusão uma lesão do interior do seio frontal esquerdo, compatível com osteoma. Com formação medindo cerca de $5,1 \times 2,0 \times 2,8 \mathrm{~cm}$ com maiores diâmetros transverso $X$ anteroposterior $x$ craniocaudal. De efeito expansivo, com compressão e desvio caudal do teto orbitário deste lado. Compressão do músculo reto orbitário superior esquerdo, com rebaixamento do globo ocular do mesmo lado e ligeiro estrabismo divergente. Também relatado espessamento mucoso no seio maxilar direito e trabeculado etmoidal. Sendo encaminhada ao otorrinolaringologista. Ao exame físico otorrinolaringológico, presente abaulamento em região frontal esquerda, indolor a palpação, de superfície lisa e sem sinais flogísticos, não apresentando dor a palpação de seios da face, linfonodomegalias ou outras alterações. Foi solicitado exame de Tomografia de seios da face para melhor elucidação do quadro e o mesmo demonstrou que o osteoma havia invadido a linha média com extensão para o seio frontal contralateral e inferiormente para o teto da órbita esquerda, evoluindo para uma leve proptose ocular ipsilateral. Sinusopatia maxiloetmoidal direita e frontal bilateral com obliteração do complexo ostiomeatal direito, podendo estar relacionado a agudização. Sendo iniciado tratamento e solicitado nova tomografia para avaliar a evolução com o tratamento proposto, com medicação sintomática, por via oral. $\mathrm{Na}$ consulta de retorno obteve leve melhora das queixas de cefaleia e ao exame de tomografia de seios da face de Maio deste ano, como resultado não se observou alterações evolutivas significativas, com piora do quadro sinusal, onde relatado sinusopatia maxilofrontoetmoidal bilateral, com obliteração do complexo óstio-meatal direito. A paciente segue em acompanhamento otorrinolaringológico e com proposta 
cirúrgica de ressecção de osteoma de seio frontal com a equipe de neurocirurgia de serviço de referência.

\section{DISCUSSÃO}

Define-se osteoma como um tumor benigno que acomete predominantemente os seios da face, caracterizado na literatura por seu crescimento lento e assintomático, podendo levar até anos para início de manifestações (BALIEIRO et al. 2004) (FERREIRA, 1992). Nesse contexto, ainda que o osteoma que acomete o seio frontal seja mais frequente, correspondendo a $57 \%$ dentre todos os paranasais, é também geralmente descoberto acidentalmente devido a sua discrição em evolução e sintomas (OLIVEIRA et al., 2002) (JOÃO et al., 2014).

Quanto à clínica, é importante ressaltar que a partir do sítio de origem em seio frontal, são descritos sintomas como cefaléia frontal, rinorréia com característica mucopurulenta (TIAGO et al., 2002). Ainda pode haver outro grupo de sintomas, os infecciosos, a exemplo das sinusites e meningites, e também alterações oftalmológicas quando os limites do osso frontal são ultrapassados, como a diplopia, ptose palpebral, epífora, diminuição da acuidade visual, proptose entre outras (FERREIRA; RIBEIRO, 1992) (TIAGO et al., 2002) (FILHO; MOYSÉS, 1951).

Este caso é notável, pois a paciente apresentava queixa de abaulamento em região frontal da face à esquerda com crescimento rápido, de início assintomático e progredindo com cefaleia, sem outros sintomas.

Sob análise epidemiológica, estudos mais recentes apontam a incidência de osteoma de seio frontal maior entre indivíduos na segunda década de vida, sendo que o diagnóstico é geralmente tardio (CARVALHO et al., 2008). Assim, corroborando com essa estatística, a paciente relatada é jovem, de 20 anos, e o diagnóstico precoce é provavelmente justificado pelo caráter progressivo e súbito da lesão.

Ademais, para conclusão diagnóstica, exames de imagem são de suma importância, pois avaliam a lesão, bem como seu tamanho, localização e extensão (TIAGO et al., 
2002). O método considerado padrão ouro é a Tomografia Computadorizada, mas pode ser identificado também em Radiografia simples de crânio e Ressonância Magnética (PEREIRA et al., 2000), como no caso descrito. O tratamento consiste em ressecção cirúrgica quando bem indicada e seguindo critérios para melhor prognóstico e menores taxas de complicações (BALIEIRO et al., 2004).

Imagem 1. Tomografia Computadorizada de Crânio- corte coronal.

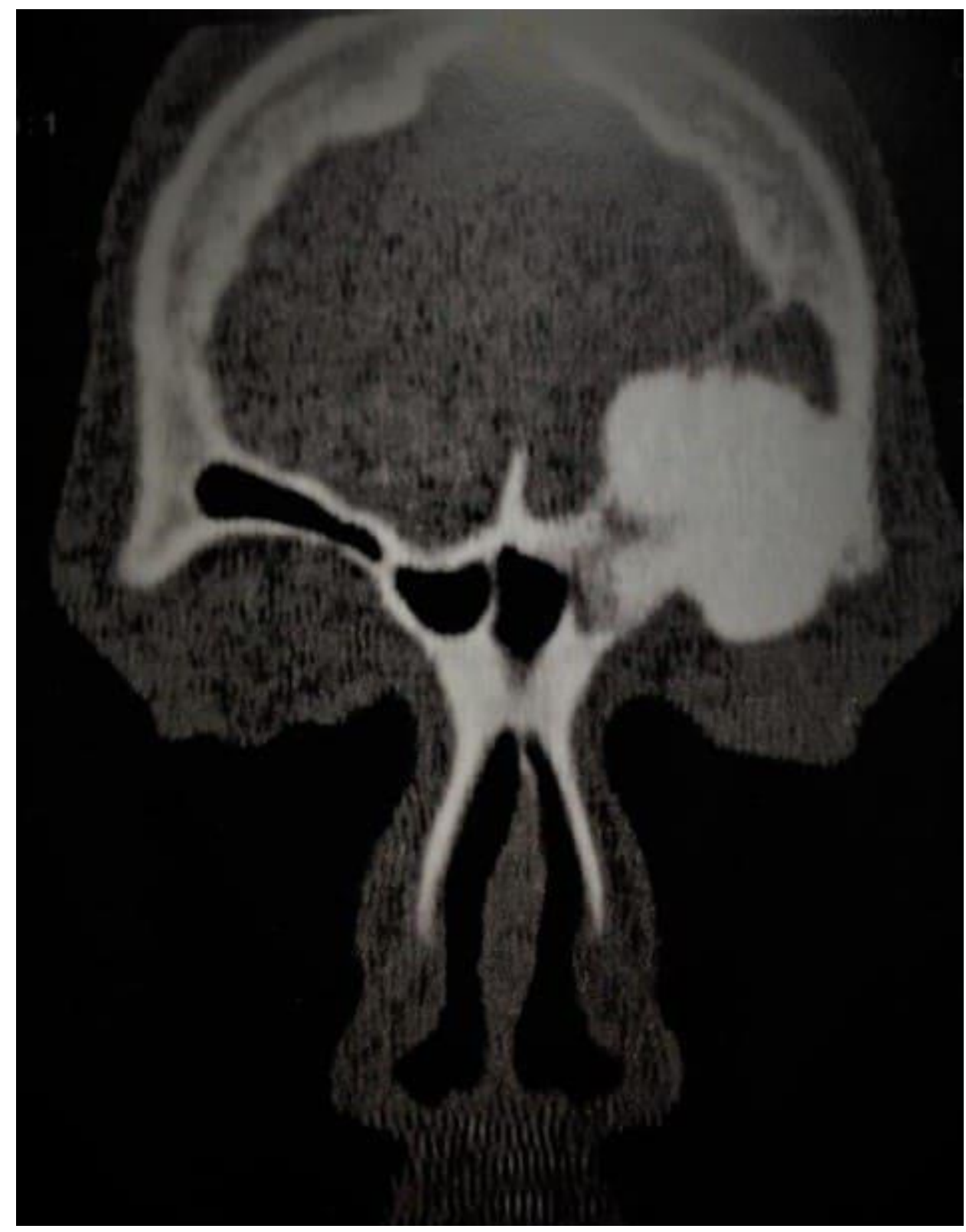

Fonte: Fotos do Autor. 
Imagem 2. Tomografia Computadorizada de Crânio- corte coronal.

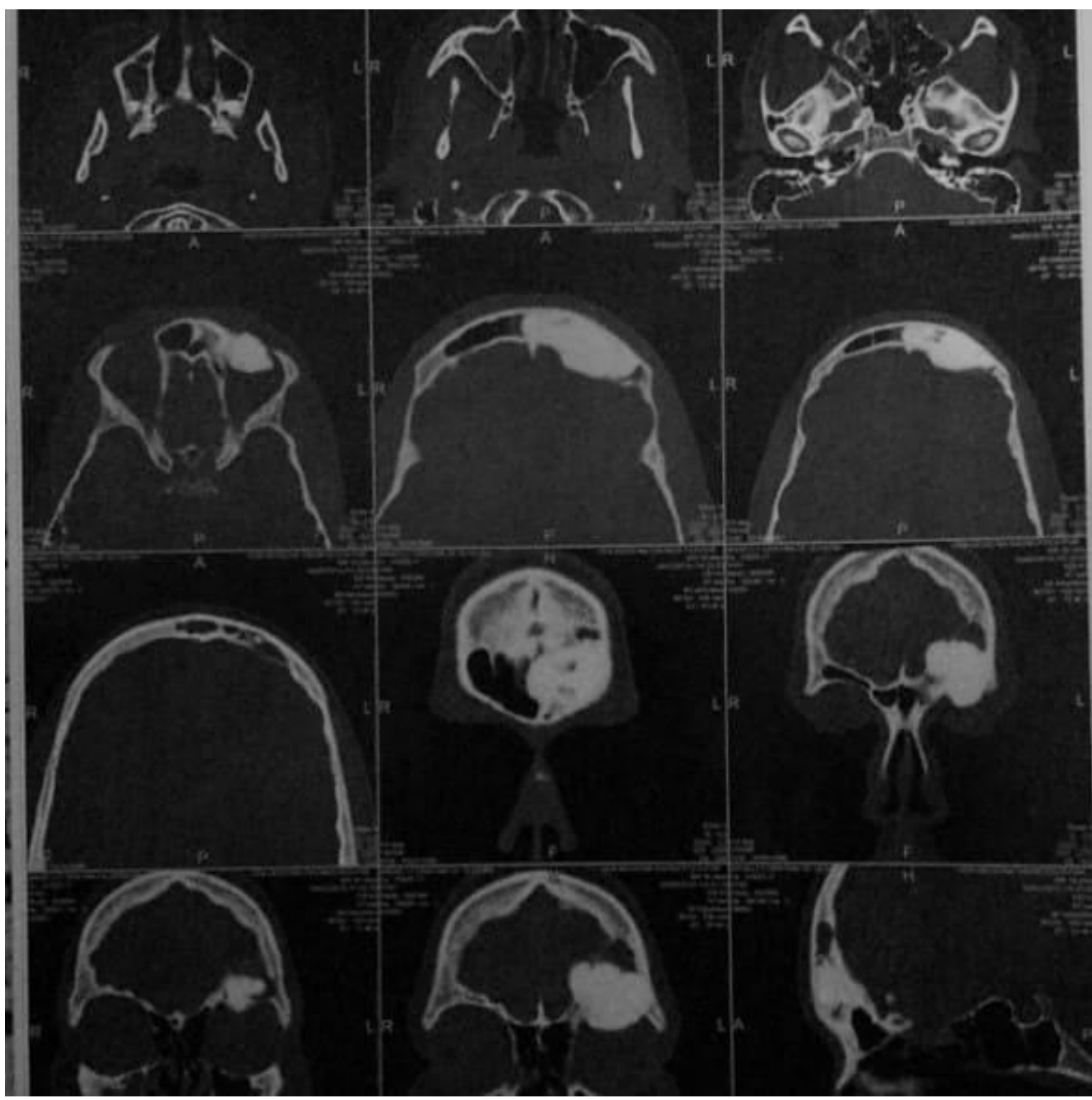

Fonte: Fotos do Autor. 


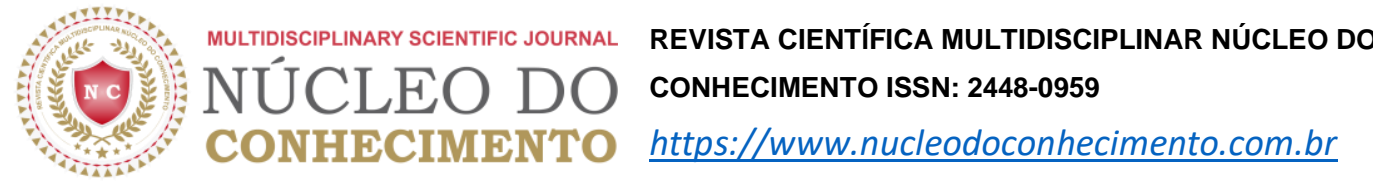

Imagem 3. Tomografia Computadorizada de Crânio - corte sagital.

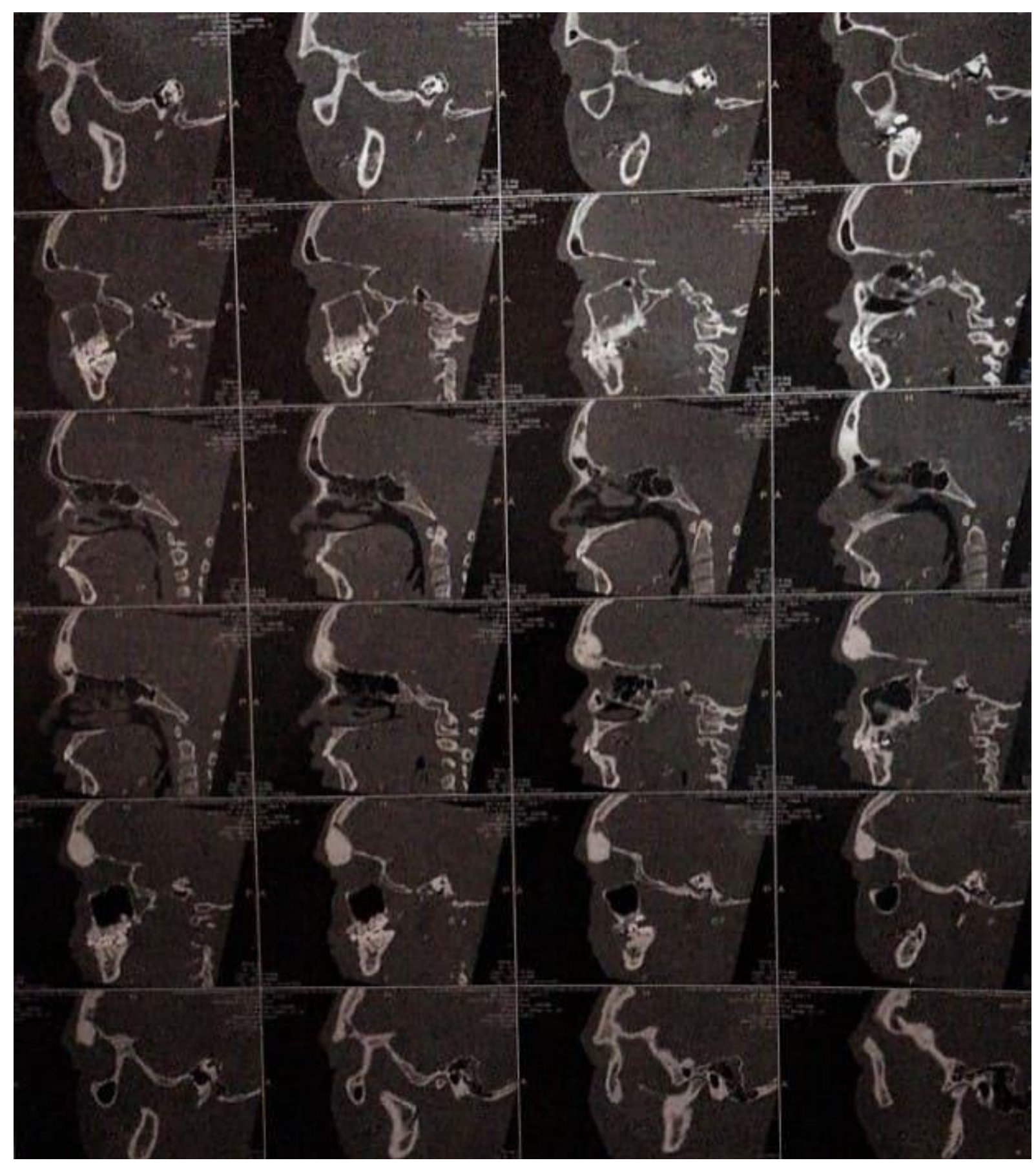

Fonte: Fotos do Autor. 


\section{CONSIDERAÇÕES FINAIS}

Sob análise epidemiológica, estudos recentes apontam a incidência de osteoma de seio frontal maior entre indivíduos na segunda década de vida, corroborando com a paciente relatada. Na literatura os estudos apontam para um crescimento lento, assintomático e de difícil diagnóstico. No entanto no caso relatado, o osteoma apresentou crescimento súbito, progressivo e sintomático, possibilitando um diagnóstico precoce.

\section{REFERÊNCIAS}

BALIEIRO, F. et. al. Abordagens cirúrgicas para os osteomas dos seios paranasais. Revista Brasileira de Otorrinolaringologia, São Paulo, v. 70, n.2, p. 164170, março/abril. 2004.

CARVALHO, R. et. al. Osteoma craniofacial: Estudo de 35 casos. Revista Brasileira de Cirurgia de Cabeça e Pescoço, v. 37, n.4, p.212-214, outubro/dezembro. 2008.

FERREIRA, A; RIBEIRO, C. Osteomas do seio frontal. Revista Portuguesa de Estomatologia e Cirurgia Maxilofacial, v.33, n.1, p.41-43, março. 1992.

FILHO, R; MOYSÉS, C. Complicações neurológicas por osteomas dos seios paranasais: A propósito de um caso de pneumatocele extradural por osteoma do seio frontal. Arquivos de Neuro-Psiquiatria, São Paulo, v.9, n.2, p.133-143, Junho. 1951.

JOÃO, L. et. al.Osteoma gigante do seio frontal - A propósito de um caso clínico. Revista Portuguesa de Otorrinolaringologia-Cirurgia de Cabeça e Pescoço, v.52, n.4, p.239-42, dezembro. 2014.

OLIVEIRA, F. et. al. Cirurgia de osteoma de seio frontal. Arquivos de neuroPsiquiatria, São Paulo, v.60, n.1, p.101-105, março. 2002. 
PEREIRA, C. et. al. Osteoma Craniano: Considerações sobre 8 casos. Arquivos Brasileiros de Neurocirurgia, Sergipe, v.19, n.4, p.170-173, novembro. 2000.

TIAGO, S. et. al. Osteomas fronto-etmoidais: aspectos clínicos e cirúrgicos. Revista Brasileira de Otorrinolaringologia, São Paulo, v.68, n.4, p.516-520, julho/agosto. 2002.

Enviado: Junho, 2020.

Aprovado: Setembro, 2020. 